\title{
La investigación escolar para la vida: diseñando nuevas experiencias formativas
}

\section{School Research for Life: Designing New Formative Experiences}

\author{
Jorge Eduardo Urueña López \\ Universidad de Antioquia \\ Calle 67 No. 53-108, 050010 \\ Medellín \\ jorge.uruena@udea.edu.co \\ ORCID ID 0000-0001-8068-952X \\ Colombia
}

\section{Resumen:}

En la actualidad, la investigación se ha convertido en una actividad necesaria y pertinente para la formación de las nuevas generaciones. Hoy, las prácticas pedagógicas se desarrollan como actividades experienciales basadas en diseños teóricos y metodológicos alternativos, como la Comunicación para el Desarrollo (Gumucio, 2001 y 2011) y el Diseño basado en Experiencias (Press y Cooper, 2009). Estas (re) significan el lugar de los estudiantes, quienes se afianzan cada vez más como investigadores que llevan al escenario educativo sus experiencias como puntos de diálogo y reflexión sobre el cómo -más allá del qué- se debe aprender para vivir en comunidad. Desde un enfoque innovador, se presentan los casos de éxito en prácticas educativas atravesadas por fenómenos de violencia urbana y sicarial en la comuna 8 de la ciudad de Medellín, Colombia, con el fin de comprender cómo el espíritu inquieto y la capacidad de asombro de los jóvenes de esta comuna, les permiten transversalizar sus experiencias de vida, de manera flexible e integral, como formas de conocimiento que deben abordar en sus escuelas. Este artículo presenta una forma que permite comprender el sentido de la didáctica para vivir en tiempos de crisis y para experimentar la vida como un aprendizaje.

Palabras clave: Investigación escolar; experiencia formativa; comunidad; comunicación; educación.

\begin{abstract}
:
Currently, research has become a necessary and relevant activity for the formation of the new generations. Today, pedagogical practices are developed as experiential activities based on alternative theoretical and methodological designs, such as Communication for Development (Gumucio, 2001 and 2011) and Design based on Experiences (Press y Cooper, 2009). These resignify the place of the students as researchers who take their experiences to the educational scene as points of dialogue and reflection on how-beyond what- they must learn to live in community. From an innovative approach, I present the cases of success in educational practices traversed by phenomena of urban and sicario violence in the commune 8 of the city of Medellin, Colombia, in order to understand how the restless spirit and the capacity for wonder of the young people from this commune allows them to use their life experiences, in a flexible and integral manner, as forms of knowledge that they must address in their schools. This article presents a way to understand the meaning of didactics to live in times of crisis and to experience life as learning.
\end{abstract}

Keywords: School research; formative experience; community; communication; education.

Recibido: 13/05/2019 | Aceptado: 09/06/2019 | Publicado: 18/06/2019

| pág. $47-69$ 
La investigación escolar para la vida: diseñando nuevas experiencias formativas ${ }^{1}$

\section{Introducción}

Partiendo del reconocimiento del diseño educativo como un área proyectual, se reconoce que el oficio del educador parte de la aprehensión del sistema ideológico en el que se inserta. Históricamente se le ha concedido al diseño educativo el lugar del oficio para responder a las necesidades 0 requerimientos de quien los suministra: en algunos casos el Estado y, en otros, la entidad privada. Algunos investigadores se han acercado a este problema desde una mirada sociológica crítica, tales como Moreno Mínguez y Carrasco Campos (2014), al asumir la educación como una disciplina que históricamente ha presentado variaciones en sus estructuras conceptuales, partiendo del reconocimiento de que dichas variaciones son motivadas por hechos históricos con bases ideológicas concretas, como la modernidad y la priorización del sistema formal para la producción.
La educación sigue pensándose como un proceso transformador de vidas, una práctica que se cuestiona a sí misma según las relaciones de sentido construidas entre el maestro, el estudiante y la comunidad bajo la lógica del sistema social y cultural -neoliberal- en el que se constituye, se legitima y actualiza de forma permanente. Aun así, no pierde su condición procesual y crítica, esa misma que la hace reflexiva al sistema de formación en competencias contemporáneo, y a partir del cual se retoman los procesos de producción y consumo que han sostenido al neoliberalismo como sistema imperante y base de la formación cultural etnocentrista en la que vive occidente. Por lo tanto, será la investigación escolar aquel camino que recorran tanto educadores como estudiantes con el cual se constriñan los intereses del mismo fenómeno cultural que 
la promueve (neoliberalismo), haciendo que el estudiante aprenda a partir de sus experiencias de vida y transformando, para el caso de Medellín, su contexto en medio de la violencia. Moreno Mínguez y Carrasco Campos (2014) lo plantean de la siguiente manera:

Si partimos del hecho de que educar es sinónimo de socializar tenemos que remitirnos al concepto de cultura. Geertz (1987) define la cultura como la trama de significaciones que el hombre ha construido en donde conforma y desarrolla su personalidad y conducta. Por lo tanto, se trata de un mecanismo adaptativo básico compuesto por un conjunto de valores y normas que junto con las prácticas y hábitos heredados y somatizados conforman un repertorio de respuestas a problemas e incertidumbres (Moreno Mínguez y Carrasco Campos, 2014, p. 236).

Pensar la investigación escolar como un escenario de posibilidades que cuestionen la institucionalización del currículo según las lógicas de sistema de producción, permite que las entidades públicas que administran la ciencia en el país consideren que la educación es un campo idóneo para la producción de conocimiento desde las aulas de clase en primaria y secundaria. Esta discusión se lleva a cabo a propósito de la incorporación de productos de la investigación y creación que han sido definidos por el Departamento Administrativo de Ciencia, Tecnología e Innovación Colciencias (Colombia) para la catalogación, donde la práctica de aula entre el maestro y estudiante de la escuela son generadores de conocimiento científico, situación que permite el reconocimiento de grupos de investigación, desarrollo tecnológico o de innovación en las áreas de las Ciencias de la Educación, Ciencias Humanas y Ciencias Sociales como referentes de ciencia. Igualmente, esta confianza depositada en la investigación escolar explica el surgimiento de los programas y facultades de educación con enfoques en las artes integradas o la innovación social, con los cuales se busca transformar las realidades de la comunidad como necesidades priorizadas por el currículo en la escuela. Asumir la ciudad como un currículo que enseña y se aprende para la vida (Cuervo, 2013). La 
investigación escolar para esta comunidad en la ciudad de Medellín se convierte en un escenario de posibilidades comunicativas con las cuales se agencian cambios en el direccionamiento curricular de cómo enseñar dentro y fuera de las aulas, convirtiendo fenómenos, tales como la violencia y el sicariato, en temas que se buscan interpelar a partir reflexión para aprender a leer, a escribir y sentir el entorno en el que vivimos: una forma de resignficar los escenarios de vida.

Es por ello que se plantea una alternativa de trabajo teóricoconceptual, donde quien transforma las prácticas culturales, de valor y sentido para la comunidad es el estudiante, aquel sujeto-objeto de la investigación en la comunidad, y quien decide llevar al aula de clase los fenómenos que transcienden en las relaciones entre vecinos, padres de familia y ciudadanos que transitan por estas construcciones de poder llamadas territorios de la 8 (Urueña, 2015), para así rediseñar la práctica educativa y comprender el valor que ésta tiene para los miembros del aula y la generación de conocimiento a partir de la experiencia permanente.

\section{|El diseño de las experiencias educativas: una forma de resignificar el espacio de vida}

En la comuna 8 de la ciudad de Medellín se vienen liderando diferentes propuestas de comunicación comunitaria, organizadas bajo la dinámica de colectivos y asociaciones juveniles que buscan el bienestar de la comunidad.

En el caso del Barrio Olaya Herrera, se ha logrado generar espacios de disertación y diálogo fraterno a partir del Centro de Investigación Social: Diseñando la Ciudad. Para la creación y desarrollo del Centro se contó con el apoyo de la Secretaría de Educación de Medellín y la Institución Educativa Barrio Olaya Herrera, en donde sus estudiantes comenzaron a aproximarse, de manera crítica y reflexiva, a los fenómenos socio-culturales que se observan en el barrio: sicariato, pandillas, vacuneros, ausencia de autoridad, inasistencia alimentaria, conflictos de género y étnicos. ${ }^{2}$ Esta mirada inquieta motivó a los estudiantes a formular interrogantes de investigación, 
donde su papel muy parecido al Sherlock Holmes contemporáneo, dio lugar a la indagación y revisión de los sentidos (significados) e imaginarios que traducen las prácticas cotidianas del sector.

De ahí que los estudiantes asumieran el papel de la investigación en la escuela como trasformador social y cultural de la comunidad (barrio) donde residen. Esta práctica conllevó a la Secretaría de Educación de Medellín a pensar nuevas formas de comprender y abordar el currículo de las diferentes ciencias que impactan el aula de clase, tanto así que permitió a la Institución Educativa Barrio Olaya Herrera vincular los proyectos de aula a la planeación de clases y a la gestión del Centro con la comunidad. Los estudiantes comenzaron a formular interrogantes que luego se convertirían en proyectos de investigación, integrados por más de cuatro a cinco estudiantes. En principio se comenzó con una base de 17 estudiantes y hasta el momento registra un acceso de 80 a 90 chicos que reciben formación en investigación escolar con enfoque social para el abordaje de sus respectivos interrogantes, es decir, "el Centro reúne casi el $7 \%$ de la población estudiantil de la institución educativa" (Secretaría de Educación de Medellín, 2015, p. 135).

La investigación escolar con enfoque social es una iniciativa que llevó la Secretaría de Educación de Medellín a la comunidad del Barrio Olaya Herrera en el año 2015, donde buscó transformar los problemas de acceso a la educación básica y media que ofrecía la institución a través de una mirada crítica del entorno y sus currículos de clase. Basada en la comunicación para el desarrollo (Gumucio, 2001), la propuesta consiste en que el estudiante ingresa a la institución con una pregunta sobre los fenómenos que circundan en el barrio y cómo una mirada desde la comunicación barrial puede transformar dichas realidades. Esta pregunta es revisada por los académicos e investigadores que hacen parte del Centro y los maestros de la institución educativa, para luego construir las planeaciones de clase y el trabajo de campo a partir de la temática y los objetos de conocimiento que conlleva esta.

Una vez que el estudiante aborda los conocimientos disciplinares 
necesarios para conocer su pregunta, se dispone a realizar trabajo de campo en jornada contraria a sus estudios, acompañado por los investigadores del Centro. En este momento, el estudiante conoce las formas en cómo diseñará su entorno y realidad para llevar a cabo una transformación. El trabajo de campo se toma como punto de partida para el diseño de una experiencia que implica su problema de investigación y el impacto que podría tener este en sus experiencias de vida (Press y Cooper, 2009), tanto escolar como comunitaria.

Aquí se retoma la mirada proyectual y procesual de la educación contemporánea, en donde el estudiante se convierte en el sujeto-objeto de la investigación, tal como lo afirmó Fals Borda (2009), cuando la participación en la (re) construcción del currículo y el cambio en la comunidad dependen del grado de interacción y vínculo emocional que relaciona el estudiante con su comunidad. Es una forma de aplicar la Investigación Acción Participativa (IAP) sin caer en la denominación teórico-conceptual con los estudiantes bachilleres. Ellos viven la IAP, no aprenden a recitar autores que trabajan la IAP. Diseñan sus experiencias de vida a partir de la investigación. Las manifestaciones artísticas urbanas como el graffiti, el performance y las flashmobs, han sido las iniciativas que han resultado de estos problemas de investigación escolar con enfoque social, convirtiendo así el placer de la experiencia, en este caso estética, como el ejercicio de clase con el cual cuestiona su realidad violenta $\mathrm{y}$ abyecta.

La investigación acción participante aporta a la configuración de la experiencia educativa propuesta por el estudiante-investigador. De esta manera posibilita el ejercicio integrador de la experiencia vivida con el currículo de clase, por medio del cual los estudiantes, orientados por sus docentes y empleando la integración de diversas áreas del saber, llegan a la resolución de planteamientos que ellos mismos han seleccionado entre diferentes opciones que provienen de las necesidades de una comunidad que vive en conflicto socio-político permanente. La IAP se convierte en la metodología de trabajo para el 
estudiante en su aula de clase. No se requiere de una lección dirigida o temática. La experiencia de vida del estudiante hace que este participe de manera crítica en sus formas de abordar (leer, escribir, comprender) su realidad inmediata. Dicha metodología inicia con la elección de una pregunta, tema o problema, luego se define el trabajo a seguir (asignación de compromisos a los integrantes del grupo, rastreo y lectura de los conceptos concernientes a la experiencia señalada), y luego se propone un trabajo de campo y análisis de la documentación obtenida con la cual se diseña un escenario de transformación de esta misma experiencia de vida. En esta misma línea de la investigación acción participante se puede citar:

En esta modalidad, docente $\mathrm{y}$ estudiantes indagan sobre los objetos de estudio de las diferentes disciplinas curriculares, bien sea sobre la teoría de las mismas, o bien sobre los aspectos prácticos y de aplicación o verificación de la teoría. El docente anima a los estudiantes a plantear problemas o preguntas de investigación participativa en áreas determinadas del currículo y los asesora para su desarrollo (Restrepo, 2009, p. 109).

\section{|¿Cómo fundamentar este campo de investigación disciplinar para la transformación de la comunidad a partir de la investigación escolar?}

Para comprender cómo se desarrolla la pregunta que traen los estudiantes al aula de clase, y como está se convierte en un interrogante de investigación, se hace necesario revisar los procesos comunicativos que ellos establecen con su comunidad. Aquí será relevante asumir la lectura, la escritura, los pensamientos sistémicos como las matemáticas y las ciencias naturales como escenarios de construcción social y cultural.

Dichos conocimientos se planean a partir de la experiencia del estudiante, la cual terminará por impactar el currículo.

El primer presupuesto epistémico de esta investigación fue percibir la lectura como una práctica pedagógica sociocultural, a partir 
del acercamiento con una manera de leer que pone en escena al texto (entorno social y cultural del barrio), al estudiante, a su contexto y su interacción con la cultura y con su vida. Por lo tanto, se hace énfasis en los procesos de comprensión lectora y en las implicaciones de la lectura inferencial, comprensiva y crítica para la formulación del interrogante de investigación desde la pregunta por la experiencia que se vive en el entorno. En este sentido no se lee el texto por el texto para una aprehensión del código lingüístico; se lee el texto por motivación, la ciudad como el texto para reconfigurar el currículo de clase.

Tal como lo citan Chaverra, Perea y Vergara (2018), si damos una mirada a lo que constituyen las maneras como se aborda la lectura en la escuela, podemos afirmar que, esta no se concibe como una práctica; se trata de ejercicios de decodificación y actividades de comprensión que no van más allá del cumplimiento de una tarea específica, en la que prima el análisis literal.

Es común ver que la lectura se convierte en un momento de tensiones constantes para el docente y para el estudiante.

En este instante, el primero impone lo que se debe leer y el otro se resiste a todo lo que represente esta acción. Lo cual conlleva a que, no se aborde la lectura de manera efectiva; y que, al finalizar la etapa escolar, los individuos resulten alejados totalmente de ella (Chaverra, Perea y Vergara, 2018, p. 44).

Por ello, es necesario el abordaje de la lectura desde el enfoque Sociocultural, que como lo plantea Silveira Caorsi (2013), "Desde lo Sociocultural la lectura se define como una 'práctica' porque es una acción con intención, cargada de valores variables de acuerdo con el contexto" (p.108). De ahí que, debemos tomar conciencia del papel determinante que tiene la escuela para llevar a cabo una propuesta de este tipo.

Con base en lo anterior, se requiere un enfoque en el que confluyen las prácticas pedagógicas como escenarios para la revaluación de lo que se lee, se escribe y se aprende en clase. 
La perspectiva Sociocultural ofrece una visión más global de la práctica de la lectura, que incluye elementos hasta ahora poco estudiados, como las identidades y los roles, los valores y las representaciones sociales, los usos de lo letrado o la diversidad retórica de las prácticas (Cassany, 2013, p. 39).

En segundo lugar, se recogieron aportes teóricos que permiten asumir la comunicación como desarrollo para la formación ciudadana. En este sentido, ésta se reconoce como derecho, como práctica viva, como medio de desarrollo de los individuos para ejercer la participación ciudadana.

En tercer lugar, se asumió el diseño de la experiencia como una configuración que permite desarrollar este trabajo de cambio social. Esta configuración se materializó en el Centro de Investigación Social: Diseñando la Ciudad como producto de la investigación de Jorge Urueña (2015) y a la vez se constituyó en el hilo conductor con el que se conectaron las actividades que se llevaron a cabo durante el tiempo de mediación con la comunidad del Barrio Olaya Herrera.

Finalmente, se plantean algunas reflexiones acerca de la necesidad de reconfigurar las prácticas pedagógicas de los maestros, ya que es necesario indagar y profundizar en las posibilidades que permite la enseñanza y aprendizaje de la lectura y la comunicación del barrio desde el enfoque sociocultural, especialmente en lo relacionado con poder leer más allá de lo evidente, con ser analíticos y críticos frente a lo que sucede en el contexto que nos rodea y en el mundo, así como en la necesidad de contribuir a la construcción de una sociedad más pluralista, respetuosa de la diferencia y participativa.

\section{lLa comunicación como desarrollo para la formación ciudadana}

La comunicación se presenta como un escenario idóneo donde los estudiantes viven las lecturas que han realizado sobre su comunidad. No es un impedimento el leer para vivir, por lo cual, la 
comunicación se ha designado como un segundo estadio de producción de conocimiento en el estudiante, una vez la lectura en todas sus manifestaciones ha permitido la motivación y el interés por descubrir cómo el mundo se transforma desde la acción del sujeto (estudiante).

Es una condición inherente a este, comunicar y ser ciudadano implica un aprendizaje, el cual no está desligado de lo que se vive en la escuela. Dado que, si una persona no puede comunicarse sin la (re) valorización de los textos que produce en y para su realidad, no puede llevar a cabo una experiencia de vida con la cual aprenda de la misma. Una realidad donde abunda la información por los diferentes medios de comunicación masivos e interpersonales, donde se dificulta la comprensión del mundo, y por ende ejercer una participación efectiva en su comunidad y tomar decisiones frente a la misma.

La comunicación se reconoce como un escenario de tensiones epistémicas para el currículo, pues este requiere de la identificación de sus interlocutores (estudiantes $\mathrm{y}$ maestros) como sujetos que configuran el acto de educar como experiencia de vida (Gumucio, 2011). En este punto vale la pena preguntarse lo que muchos chicos de la Institución Educativa Barrio Olaya Herrera se preguntan: “para qué estudiar si al salir de aquí las cosas en el barrio siguen de la misma manera? El que roba lo sigue haciendo y lo que el que ve robar tampoco le importa" (Comunicación personal, 20 de agosto de 2015). La comunicación se asume como aquella atmósfera con la cual se busca cambiar la pregunta del currículo tradicional. Se pasa de la clase habitual a la experiencia de vida convertida en problema de clase, de tal manera que el estudiante pasaría a preguntarse: “Cómo está leyendo el ciudadano del barrio la problemática del robo, específicamente aquel que ve robar $\mathrm{y}$ no hace nada al respecto? ¿De qué manera se puede cuantificar y cualificar la práctica de robo en el barrio? ¿Existen datos o investigaciones que hablen del porqué la comunidad del barrio no hace nada ante el robo? ¿De qué manera incide el conflicto armado $y$ el narcomenudeo en la frecuencia de robos dentro del barrio?" (Comunicación personal, 25 de octubre de 2015). 
Cuando en la comunicación no se dan las condiciones que garantizan la comprensión, se recae en el ritual, en el sin sentido, en la obligación ilegítima, en el aburrimiento. La ausencia de comprensión puede deberse a múltiples factores: Es posible que el tema de la comunicación requiera de ciertos presupuestos de los que se carece. En casos como el de las matemáticas, la ausencia de un presupuesto puede ser trágica a largo plazo: quien dejó de comprender una operación o una relación fundamental, tampoco puede, en principio, comprender los desarrollos que se siguen de su aplicación. La comunicación en matemáticas puede romperse desde los primeros años de escuela, ocasionando el abandono del sistema $\mathrm{y}$ generando en el estudiante una conciencia de incapacidad de efectos impredecibles. Es posible que el lenguaje de la clase sea esencialmente distante del que se utiliza en la vida cotidiana, que las relaciones entre los significados, los contenidos a los cuales están asociados y los contextos de su uso en la escuela no se correspondan con los que tienen vigencia en la familia $y$ en el barrio, que exista una diferencia insalvable de códigos. Es posible que las fuentes de conocimiento o las formas de evidencia de los estudiantes sean muy distintas de las que presume la escuela. En cualquiera de estos casos no se cumple el presupuesto de la comprensibilidad (Hernández, 2017, p. 4).

Lo que denota que la comunicación como desarrollo para la formación ciudadana, puesto que se es ciudadano desde las comprensiones que se tienen del mundo y de la realidad, por ende, fortaleciendo la lectura inferencial se puede aportar a este tipo de formación. Tal como lo plantea Castrillón (2007) teniendo en cuenta a Gumucio, "la formación de un lector es también la formación de un ciudadano" (p.77), porque al leer, se adquieren elementos de comprensión que le permiten al sujeto tener argumentos para expresarse en la sociedad, comprender las diferentes posturas frente a las realidades de su contexto, acercarse a las problemáticas existentes $\mathrm{y}$ 
participar de manera activa en ella favoreciendo la toma de decisiones en la escuela, el barrio, la familia y la sociedad.

El andamiaje de la comunicación para la formación ciudadana demarca que, en el nivel de básica secundaria, se requiere trabajar de forma reflexiva e intencional sobre la comunicación con la que los actores de la comunidad viven día a día. Cómo se comunica el estudiante con el padre, el vecino, el líder comunitario y con los muchachos de abajo. ${ }^{3} \mathrm{La}$ comunicación de lo no dicho logra empoderar a los más pequeños para hacerlos partícipes de su contexto, de reconocerse como sujetos de derechos que le apuestan su lugar político y que desde ahí se postulan como actores de la comunidad.

Lo anterior, deja ver que la formación ciudadana, entendida tal como lo plantea el Ministerio de Educación Nacional [MEN] (2006), apunta hacia:

El desarrollo de las competencias y los conocimientos que necesitan niños, niñas y jóvenes del país para ejercer su derecho a actuar como agentes activos $\mathrm{y}$ de manera constructiva en la sociedad: para participar activa y responsablemente en las decisiones colectivas de manera democrática, para resolver los conflictos en forma pacífica y para respetar la diversidad humana, entre otros importantes, como proteger el medio ambiente (p. 154).

En este sentido el aula ha de ser uno de los espacios propicios en la institución educativa donde se geste esta dinámica de participación, de diálogo, de reconocimiento mutuo de las diferencias y de valoración de éstas. Los estándares básicos de competencia ciudadana del MEN (2006) así lo establecen y, no solo desde las áreas con que se asocia la formación ciudadana que son las ciencias sociales y ética, sino que la especifica como una tarea transversal de todas las áreas obligatorias y fundamentales que precisa la ley 115 de 1994 (Ministerio de Educación Nacional, 1994).

Por tanto, la formación ciudadana ha de ser apoyada por la transversalización de las áreas 
en los proyectos curriculares de la escuela, en sus habilidades básicas: hablar, escuchar, escribir, leer, enumerar, problematizar, solucionar, mediar, innovar, investigar, entre otras; para potenciar la habilidad comunicativa que es indispensable en el ejercicio de la ciudadanía en los términos anteriormente expresados.

La transversalización de saberes disciplinares se contempla como un escenario de acciones educativas encaminadas a entender las necesidades de la comunidad a través de las temáticas que privilegia el currículo. Se toma como el reto ontológico que debe asumir la educación como una práctica que no responde solo a la mirada institucional basada en el sistema de producción neoliberal, sino a su esencia como práctica pedagógica con la que se cuestiona la forma de aprender y enseñar en un contexto mediado por la producción en sí. Se investiga desde la transversalización de saberes disciplinares no para responder al mercado laboral e innovar en el mismo, por el contrario, se investiga para transformar las realidades de la comunidad y la escuela atravesadas por el fenómeno que le agobia: el conflicto armado.

Se entiende la transversalización como la propuesta de estándares que integra las competencias con conocimientos básicos, procesos generales y contextos; por consiguiente, no se presentan como un listado detallado de contenidos ni como una serie de conceptos o procesos aislados. Una de las características más sobresalientes es su transversalidad y la intencionalidad de dotar de significado esta relación (Ministerio de Educación Nacional, 2004, p. 172).

Es por eso que, la comunicación entre la escuela y el entorno debe permitir dicha transversalización, la cual posibilite el tránsito de los imaginarios que se construyen en la comunidad. Pasar del estudiante al ciudadano sin perder de vista los intereses de formación y las lógicas de la escuela contemporánea, como también movilizarse del lugar del muchacho de abajo (Urueña, 2015) al estudiante que propone cambios en la comunidad. 
|El diseño de la experiencia como una configuración que permite desarrollar este trabajo de cambio social

Llegar a pensar el currículo escolar como un diseño de la experiencia del estudiante, en calidad de ciudadano, ha sido una de las apuestas epistémicas más relevantes del actual proyecto de investigación. El diseño es la organización de acciones de enseñanza orientadas al aprendizaje, a las características de la interacción, los discursos y materiales de soporte que garanticen la mediación del estudiante con el entorno a través del paso de una pregunta coloquial por su comunidad al interrogante de investigación.

Se considera el diseño una configuración que favorece el análisis de la práctica pedagógica, tal como lo expresa $E l$ diseño como experiencia: El papel del diseño $y$ los diseñadores en el siglo XXI (Press y Cooper, 2009). La experiencia de vida, al ser tomada como referente para la transformación del currículo de clase, se convierte en la materia prima para la gestión de sus respectivos proyectos de investigación en la comunidad. A partir de ella se diseñan formas de comunicación e interacción con el entorno que impliquen liderazgo y transformaciones por parte de las chicas y chicos que residen allí mismo.

Así mismo, como lo plantea Press y Cooper (2009) "La estructura de la experiencia se integra con dos elementos que se realizan de manera paralela: la secuencia de las actividades para el aprendizaje y la evaluación de cómo se aprende en el contexto" (p.4). Lo que quiere decir que, es un proceso flexible y reflexivo que permite hacer ajustes en cualquier momento de la aplicación, teniendo en cuenta la pertinencia y el impacto de ésta como un medio con el cual podemos planear las actividades de clase y cualificar los procesos de enseñanza y aprendizaje.

\section{|Reconfiguración de las prácticas de los maestros en el aula educativa de Medellín}

En la comunidad del Barrio Olaya Herrera, la transformación de los docentes ha sido permanente desde el momento en que se trabaja bajo 
la modalidad de la comunicación para el desarrollo de la formación ciudadana apremiando la comprensión del porqué se quiere aprender y formar en relación al mundo actual.

Es responsabilidad de cada maestro prever actividades e intervenciones que favorezcan la presencia en el aula del objeto de conocimiento tal como ha sido socialmente concebido, así como reflexionar sobre sus prácticas ciudadanas y efectuar las rectificaciones que resulten necesarias y posibles.

Cabe mencionar que los maestros deben garantizar escenarios de comunicación, interesar a los estudiantes para dotarlos de formas para comprender el sentido de sus realidades, situaciones que están más allá de los límites de la institución y lo instructivo como lo señala Solé (1995). Esto es lo que se pretende implementar con esta propuesta: reconocer el papel que tiene el estudiante dentro del aula de clase cuando aboga por su lugar de ciudadano, de mediador a partir de los procesos de investigación escolar con enfoque social con los que desea (re) conocer su comunidad.
|La experiencia de trabajo: El Centro de Investigación Social Diseñando la Ciudad, una experiencia significativa en el Barrio Olaya Hterrera de la ciudad de Medellín

En este Centro, los estudiantes de la Institución Educativa Barrio Olaya Herrera, institución adscrita al núcleo académico número 923 de la Secretaría de Educación de Medellín, se encuentran llevando a cabo la conceptualización e implementación de las técnicas de investigación social que se requieren para realizar una propuesta de comunicación que logre resolver didácticamente, a través de la teoría del diseño de experiencias, las necesidades que actualmente presenta el barrio en todos sus espacios culturalmente definidos.

Bajo la conceptualización del diseño y la comunicación, como campos disciplinarios emergentes que buscan contemplar la interacción social y la práctica cultural como objetos de estudio, se presenta un plan de trabajo a un año (año lectivo), en donde los estudiantes podrán acoger el fenómeno social y cultural para comprenderlo desde estas 
dimensiones teóricas y desde las implicaciones que tiene este dentro de la ciudad de Medellín. Una vez comprendido el fenómeno, y habiendo formulado un plan de gestión que se resolverá desde la comunicación, se procederá a planear el diseño de las interacciones sociales a través de la materialización escogida por el grupo de observadores.

Los propósitos de este Centro son dos puntualmente:

- Desarrollar competencias de indagación como ejercicios previos a la actividad de investigación que exigen las instituciones de educación superior (IES) acreditadas por el Ministerio de Educación y la Comisión Nacional de Acreditación (CNA), con el fin de preparar al estudiante bachiller, futuro universitario, en el eje de la investigación, innovación y desarrollo social y cultural.

- Aproximar al uso de las herramientas de análisis de investigación social en la ciudad de Medellín. De acuerdo con las necesidades de los estudiantes, se reforzará mucho en el pensamiento estratégico $\mathrm{y}$ el diseño de la gestión de la comunicación y el diseño como alternativas a cambios de orden cultural, principalmente (Urueña, 2015).

La metodología de esta actividad pedagógica está basada en el trabajo de campo activo del estudiante, en donde se establecen ejercicios de aproximación en métodos de investigación social, entre los cuales, por interés de los mismos chicos y chicas, se identifican con mayor grado de aceptación la Investigación Acción Participante (IAP); de igual manera, se procede a la edificación de un escenario de clase tipo constructivista, en el cual, según Vigotsky $\mathrm{y}$ sus postulados sobre la (re) construcción del conocimiento a partir de la interacción con el medio social en el que se encuentra el individuo, se lleva a cabo la sistematización de la experiencia, con el fin de comprender cómo el o la estudiante aplica los conceptos en los interrogantes y objetivos que se ha planteado previamente sobre el fenómeno social que le interesa abordar. Para el cumplimiento de estas acciones no solo cuenta con el trabajo en clase orientado por los docentes de las respectivas áreas participantes -especialmente 
Humanidades y Ciencias Sociales-, hace a la práctica cultural que sino también con el acompañamiento de sus padres de familia, quienes, a su vez, se convierten en sujetos de las investigaciones que realizan ellas $\mathrm{y}$ ellos mismos.

Se parte de la premisa teóricoconceptual donde todo fenómeno social y cultural está basado en la construcción ideológica, de sentido, que aporta una comunidad de hablantes. A través del eje del lenguaje y, su observación desde los estudios de la comunicación para el cambio social (Gumucio, 2001), se propone una transformación del fenómeno social a través del cuestionamiento que el estudiante sustenta este hecho, dentro del aula de clase y su transformación liderada por los mismos investigadores.

Los estudiantes, como agentes del cambio, están investigando adentro y al margen de la comunidad. Los investigadores se contemplan como objetos-sujetos de la investigación. Por lo tanto, para esta actividad, se trabajan tres competencias idóneas en la formación del estudiante de básica secundaria: competencia formativa en valores, competencia investigativa social y científica, y competencia ciudadana. A continuación se presenta el modelo de trabajo del Centro:

\section{Abordaje teórico - práctico del taller}

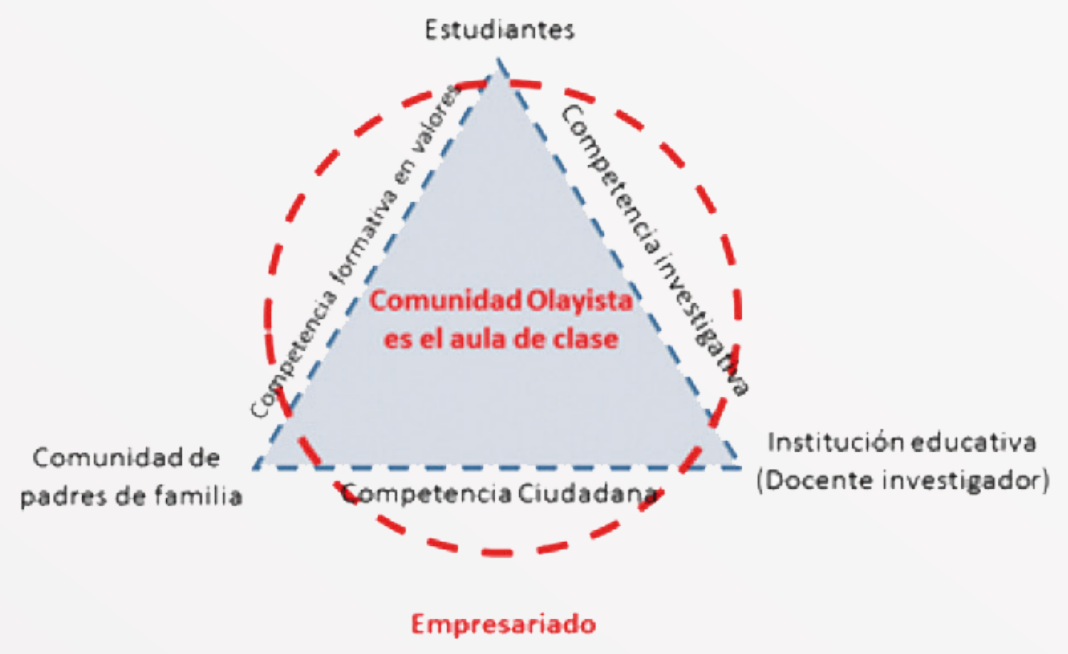

Figura 1: Abordaje teórico-práctico del Centro de Investigación Social: Diseñando la Ciudad. 
Por ejemplo, uno de los proyectos que encabezan este Centro es el Plan para el Plan, en el cual los estudiantes líderes han propuesto resolver el problema de la movilidad del barrio Olaya Herrera a través de medidas cívicas que apoya actualmente la Secretaría de Movilidad de la ciudad. El mal parqueo de los transportes motorizados, la ausencia de señalización en la única vía del barrio, las altas velocidades que presentan los carros de transporte público y la falta de zonas comunes para el tránsito peatonal, se han convertido en fenómenos de observación en el aula de clase, donde los chicos y chicas han comprendido que el problema radica en los bajos niveles de comprensión lectora-verbal que tienen los miembros de la comunidad y la preferencia de esta misma por el lenguaje visualcorpóreo. Por lo tanto, junto con la secretaría mencionada, se han propuesto señalizar cívicamente la vía, a partir de elaboración de señales de tránsito humanas en la clase de lenguaje visual y corpóreo, construyendo signos con marcos de referencia cultural encontrados en los encuentros con la comunidad.
De igual manera, han desarrollado habilidades comunicativas para el desarrollo de la asamblea ciudadana con los transportadores del barrio, donde se presentan y dialogan acuerdos y sanciones simbólicas para los transeúntes y transportadores de las calles. Se han estipulado espacios de formación con padres de familia entre la norma de tránsito, que esté en codificación verbal, y los videomanuales que los investigadores proponen para superar la crisis de información que se vive dentro de la comunidad. Jean Carlos Taborda, estudiante de séptimo grado de la institución educativa, dice: "el problema de la movilidad no solamente es un problema de planeación de la ciudad, el problema es social y cultural, es de la gente que maneja los carros" (Comunicación personal, 17 de agosto de 2015).

Esta experiencia podría ser replicable en diferentes niveles de formación, dado que las competencias adquiridas se pueden hallar con perspicacia y facilidad en las relaciones socio-culturales que establecen los investigadores principales, para este caso, los estudiantes de las instituciones 
educativas públicas que pertenecen a la Secretaría Municipal de Medellín. Actualmente se dispone de tres líneas de investigación donde se abordan seis fenómenos socio-culturales que se presentan en el barrio:

- Estudios culturales urbanos. En esta línea, estudiantes y maestros se acercan a pensar los derechos, las diferencias y la diversidad como procesos de formación cultural a partir de ejercicios simbólicos, con los cuales se comprende al otro como a sí mismo. Una forma de construir identidades a partir de las narrativas del sujeto en cuestión: el estudiante. En esta línea se encuentran registrados proyectos como Cultura Afro, donde este espacio de diálogo gira en torno a las narrativas de los habitantes del barrio como formas de (re)existencia y apropiación en un lugar políticamente segregado entre "paisas, negros y venezolanos". ${ }^{4}$

- Estudios medio-ambientales y civilidad. En este trayecto, la comunidad se asume como un laboratorio de experiencias en ciudadanía con las que el

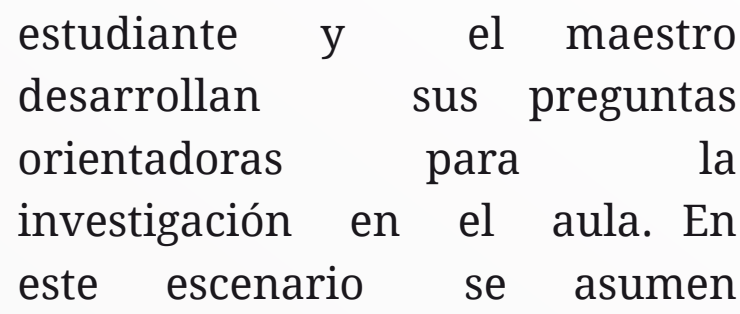
problemáticas de la comunidad en términos de infraestructura, señalización vial y manejo de residuos orgánicos como fenómenos que culturalmente pueden ser reconfigurados semióticamente a través del lenguaje, la comunicación y la resolución de problemas. Los proyectos que se registran en esta línea son: El Plan para el Plan: estrategia de comunicación para intervenir la movilidad y el tránsito en el Barrio Olaya Herrera y, La basura se organiza en casa: proyecto social para la recolección de residuos orgánicos como formas de abastecimiento de huertas escolares.

- Estudios en salud y bienestar social. En esta línea, el abordaje de investigación tiene como principal motivación el bienestar de las personas. La salud se comprende como un fenómeno que cultural y socialmente se aprende y se enseña en todas las instancias de la vida: el hogar, 
la calle, el barrio, los colectivos juveniles y por supuesto la escuela. Este escenario posibilita que el maestro, de las áreas de las ciencias naturales y matemáticas, contemple el conocimiento disciplinar como una forma de leer y diseñar a partir de la experiencia, el currículo de la clase como práctica ciudadana. Los proyectos que se registran en esta línea son: PAC: Plan de Alimentación Ciudadana en el Olaya Herrera, propuesta de comunicación para transformar la nutrición en el barrio y, Ahorradores de energía: Propuesta de comunicación para la transformación en el consumo excesivo de energía dentro del barrio Olaya Herrera.

\section{|A manera de conclusión}

De este proceso de reconfiguración de la práctica educativa en el aula de clase, quedan dos puntos importantes a reconocer:
- El diseño de la experiencia aborda algún (o algunos) procesos de la comunicación y el lenguaje, generalmente ligados a una realidad materializada en los currículos de clase. En el diseño se encuentran las pistas para repensar el currículo contemporáneo, dado que el diseño suministra al maestro investigador las herramientas para transformar su didáctica, en términos de forma, desde la experiencia del estudiante. El maestro se moviliza hacia el cambio, no en su condición de maestro, sino en su lugar de ciudadano; tal como lo hacen los estudiantes.

- El conocimiento se metaforiza en el sentido que trasciende de su dimensión sistémico-cognitiva y comienza a destacarse por su dimensión experiencial, donde el aula se convierte en un escenario de diversos interrogantes que sirven para orientar el proceso formativo tanto del estudiante como del maestro. 
La investigación escolar para la vida: diseñando nuevas experiencias formativas

Urueña López

\section{|Notas al final}

1 El presente artículo es resultado del proyecto de investigación denominado Centro de Investigación Social: Diseñando la Ciudad de la Institución Educativa Barrio Olaya Herrera (Núcleo académico 923) Secretaría de Educación de Medellín (Colombia) en los años 2015-2017.

2 Citados así por los registros y bitácoras de trabajo de campo del Centro de Investigación Social: Diseñando la Ciudad de la Secretaría de Educación de Medellín (Colombia).

3 Tomado de los diarios de encuentros del Centro con la comunidad del barrio Olaya Herrera (2015).

4 Expresiones tomadas de los diarios de encuentros del Centro con la comunidad del barrio Olaya Herrera (2015). 


\section{|Referencias}

Cassany, D. (2013). Prácticas letradas contemporáneas. Recuperado de https:// bit.ly/2I6pkBG

Castrillón, S. (2007). Alfabetización, ciudadanía y toma de conciencia. Recuperado de https://bit.ly/2KGWBoP

Chaverra, I., Perea, N. \& Vergara, L. (2018). La lectura inferencial: punto de referencia para la formación ciudadana en la escuela. Medellín, Colombia: Universidad de Antioquia.

Cuervo, M. E. (2013). Currículum, ciudad y formación de maestros en contextos de violencia urbana. Temas de Educación, 19(1), 135-148. Recuperado de https://revistas.userena.cl/index.php/teduacion/article/view/384

Fals Borda, O. (2009). El conflicto, la violencia y la estructura social colombiana. Bogotá, Colombia: Siglo del Hombre Editores CLACSO.

Gumucio, A. (2001). Communication for Social Change Anthology: Historical and Contemporary Readings. New York, NY: NYC University Press.

Gumucio, A. (2011). Comunicación para el cambio social: clave del desarrollo participativo. Revista Signo y Pensamiento, 58(30), 238-253.

Hernández, C. (2017). Educación y comunicación: pedagogía y cambio cultural. Nómadas, 5, 1-20

Ministerio de Educación Nacional. República de Colombia. (1994). Ley 115 de 1994. Bogotá, Colombia: Ministerio de Educación Nacional.

Ministerio de Educación Nacional. República de Colombia. (2004). Transversalidad de los estándares básicos de competencias. Bogotá, Colombia: Ministerio de Educación Nacional.

Ministerio de Educación Nacional. República de Colombia. (2006). Estándares Básicos de Competencias en lenguaje, Matemáticas, ciencias y ciudadanas. Bogotá, Colombia: Ministerio de Educación Nacional. 
Moreno Mínguez, A. \& Carrasco Campos, A. (2014). El papel de la sociología en las ciencias de la educación: aportaciones y competencias para los futuros maestros. TABANQUE Revista pedagógica, 27(2014), 235-250.

Press, M. \& Cooper, R. (2009). El diseño como experiencia. El papel del diseño y los diseñadores en el siglo XXI. Barcelona, Españal: GG Diseño.

Restrepo, B. (2009). Investigación de aula: formas y actores. Educación y Pedagogía. 21 (53), 103-112. Recuperado de https://bit.ly/2K8Wkvh

Secretaría de Educación de Medellín (2015). Informe de rendición de cuentas del periodo gubernamental 2011-2015. Medellín, Colombia: Universidad de Antioquia.

Silveira Caorsi, E. (2013). La lectura como práctica sociocultural y herramienta para lograr la equidad social a partir de la enseñanza. Revista Cuadernos de Investigación Educativa, 4(19), 105-113. Recuperado de https://bit. ly/2KH5gaI

Solé, I. (1995). El placer de leer. Lectura y Vida. Revista Latinoamericana de Lectura. 16 (3), 2-8. Recuperado de https://bit.ly/2IxDESV

Urueña, J. (2015) La investigación escolar como la transformación del barrio desde un enfoque de la comunicación para el cambio social. Medellín, Colombia: Programa Ondas Colciencias - I. E. Barrio Olaya Herrera.

Vygotsky, L. (1978), Pensamiento y lenguaje. Madrid, España: Paidós. 